\title{
Hydration Dynamics and the Future of Small-Amplitude AFM Imaging in Air
}

\author{
Sergio Santos ${ }^{1, *}$, Tuza A. Olukan ${ }^{1}$, Chia-Yun Lai ${ }^{1}$ and Matteo Chiesa ${ }^{1,2}$ \\ 1 Department of Physics and Technology, UiT The Arctic University of Norway, 9037 Tromsø, Norway; \\ tuza.a.olukan@uit.no (T.A.O.); chiayunlai@gmail.com (C.-Y.L.); matteo.chiesa@uit.no (M.C.) \\ 2 Laboratory for Energy and NanoScience, Masdar Institute Campus, Khalifa University of Science and Technology, \\ Abu Dhabi 127788, United Arab Emirates \\ * Correspondence: ssantos78h@gmail.com
}

check for

updates

Citation: Santos, S.; Olukan, T.A.; Lai, C.-Y.; Chiesa, M. Hydration Dynamics and the Future of Small-Amplitude AFM Imaging in Air. Molecules 2021, 26, 7083. https:// doi.org/10.3390/molecules26237083

Academic Editor: Marek Kosmulski

Received: 7 October 2021

Accepted: 18 November 2021

Published: 23 November 2021

Publisher's Note: MDPI stays neutral with regard to jurisdictional claims in published maps and institutional affiliations.

Copyright: (c) 2021 by the authors. Licensee MDPI, Basel, Switzerland. This article is an open access article distributed under the terms and conditions of the Creative Commons Attribution (CC BY) license (https:// creativecommons.org/licenses/by/ $4.0 /)$.

\begin{abstract}
Here, we discuss the effects that the dynamics of the hydration layer and other variables, such as the tip radius, have on the availability of imaging regimes in dynamic AFM-including multifrequency AFM. Since small amplitudes are required for high-resolution imaging, we focus on these cases. It is possible to fully immerse a sharp tip under the hydration layer and image with amplitudes similar to or smaller than the height of the hydration layer, i.e., $\sim 1 \mathrm{~nm}$. When mica or HOPG surfaces are only cleaved, molecules adhere to their surfaces, and reaching a thermodynamically stable state for imaging might take hours. During these first hours, different possibilities for imaging emerge and change, implying that these conditions must be considered and reported when imaging.
\end{abstract}

Keywords: AFM; small amplitude; hydration; water; multifrequency

\section{Introduction}

The atomic force microscope can be operated in air, ultra-high vacuum (UHV), and liquid environments [1]. Nevertheless, a multiplicity of phenomena controlling cantilever dynamics [2], modes of operation [3], and feedback mechanisms [4,5] influence the possibilities and challenges encountered in each environment. This calls for attention in terms of the operation, utilization, and interpretation of data; for this reason, different theories have been developed and implemented in many cases [6]. The specific properties and morphology of samples [7], such as elasticity or viscoelasticity, [8] must also be considered when operating the instrument. Moreover, the user must cope with and understand the character of some dynamic variables, such as the sharpness of the tip [9,10], otherwise referred to as the tip radius, $R$, in high-resolution imaging applications. Perhaps counter-intuitively to the newcomer, the field has rapidly advanced in two extremes-in liquid [11-14] and UHV environments [15]—while several complex phenomena have hindered the imaging and quantification of phenomena in air [16] with similar resolutions, controls, or throughputs [3]. There has been research in air in terms of capillary interactions [17], spontaneous capillary condensation [16], and the way the air environment affects surfaces [18], molecules on it, and modes of imaging [3,19-21]. In terms of force measurements in air, most have focused on capillary interactions [22] arising from the formation and rupture of a capillary neck as a function of the relative humidity (RH), either experimentally [16] or theoretically [23]. Here, a capillary neck between the tip and the substrate forms and ruptures. In dynamic AFM, it is argued that the neck forms and ruptures [22,24] during each oscillation cycle, implying that the process is stable enough to occur at a rate of thousands of times per second. Some models consider the constant volume approximation [25], while others argue that the condensation from water in the neighborhood of the tip sample junction is also responsible for the volume of the neck [16]. The constant volume approximation assumes that the water layers on the surfaces contribute to the neck. Some have produced 
models to predict the values of formation and rupture of the neck at distances $d_{\text {on }}$ and $d_{\text {off }}$, respectively [22].

Force reconstruction techniques in air are currently being employed to decouple oscillatory and monotonic terms and simultaneously resolve interfacial water structures in liquid with an atomic resolution [12]. The oscillatory contributions correspond to the surface forces that provide information about the spatial frequencies of the liquid density. The monotonic term, many times neglected by the community developing the 3D mapping force technique [16], is the component of the force typically studied by force reconstruction methods that rely on averaging. It is worth noting, in the context of the topic under consideration, that even current research tends to focus on [26] the distinction between dry conditions and high-humidity conditions, i.e., extremes, and might obviate the aging process of the surface [27]. In short, while extremes, i.e., dry conditions and high relative humidity (RH) conditions, are sometimes considered by the community, in 2018, some [18] emphasized that the dynamic processes occurring on the surfaces due to nanoscale contamination are still largely neglected. This is even the case when the state of the surface is known to affect properties such as conductance [28], and research on surface contaminants and the quantification of interfacial properties in air is ongoing [29]. Arguably, less consideration has been paid to the phenomena arising from hydration dynamics and surface contamination as surfaces age [30] in relation to the alternatives that emerge for imaging. That is, adsorption kinetics is a process that occurs in time, and this can result in the emergence or absence of different imaging modes [21,31] also in time. Here, we explicitly interpret the problems that can arise when imaging in air due to the dynamics or adsorption kinetics of water molecules and other surface adherents. Previously, we have discussed the different regimes that are available when imaging in air with small amplitudes [32,33] in two imaging extremes, i.e., fresh and ambient-aged samples in air environments. We have also discussed the variations of force measurements as a function of time $[27,34]$ and the effects on AFM measurements due to the presence of water films on surfaces [35]. The latter line of research provides information about the evolution of force profiles, but the connection between such evolution and the emergence of force regimes is stated mostly, or only, with respect to extreme conditions, i.e., fresh samples and aged samples.

In this work, we emphasize that the connection between the understanding of hydration dynamics, or adsorption kinetics, of contaminants such as water gained from force measurements and the possibilities for imaging is still largely lacking. Since these two objects of investigation are connected, it should be possible to understand how and when they affect each other. Thus, in this brief article, we discuss possible ways to connect the analysis of force reconstruction in time and as surfaces age on the one hand, and the evolution of imaging regimes in air and the possibilities that these offer on the other.

\section{Discussion}

\subsection{The Problem of Small Amplitude Imaging and Surface Aging}

The recent work by Eichhorn and Dietz [3] is particularly suitable to investigate the problems that might emerge when (1) imaging in air with small or ultra-small amplitudes and (2) advancing the field of multifrequency AFM, where several cantilever modes are excited in multiple ways, i.e., flexural and torsional. The first point is relevant because (1) such small amplitudes are of the same order of magnitude or smaller than the water films that form on surfaces, i.e., $\sim 0.1-1 \mathrm{~nm}$, and (2) it shows that even recent research fails to report the actual regime of operation, i.e., how many regimes were available for imaging in the experiments and which regime was exploited. This may be due to the fact that research in this field is still lacking. The second point is relevant because the work is recent, state-of-the-art, and possibly indicates how high-resolution and high-throughput multifrequency methods can or should advance in the future.

The problem can be stated as follows: in their approach, the authors used ultra-small oscillation amplitudes in order to reach sufficiently small tip-sample distances and thus 
increase resolution [19]. Nevertheless, since several amplitude branches might be available depending on the condition and state of the hydration layers, the actual imaging regime that was exploited remains ambiguous. Here, reproducibility also becomes a problem, for two main reasons. First, different imaging regimes might require varying the operational parameters depending on the conditions of the surface in order to be accessed. Second, other groups might fail to reproduce the results if the imaging regimes available and the conditions of the surface are not reported together with the operational parameters that have been used. Furthermore, what small amplitude means remains arbitrary without considering cantilever, sample, and environmental parameters since the limit to stability with small amplitudes prescribes what small means [20,36]. Next, different possibilities are discussed with a view to exploiting imaging regimes in both standard dynamic AFM and multifrequency AFM in air while considering such phenomena.

\subsection{Force Reconstruction and Imaging}

Previous reconstruction experiments, as a function of surface aging, show that variations in the tip-surface force can be dramatic, even for a given tip and sample as the surfaces age in air (Figure 1). The phenomenon that we term "aging" in this work relates to the changes that occur on a surface upon exposure to the air environment [35], including the effects that varying temperature and relative humidity $(\mathrm{RH})$ might have on it over time [16]. The processes and phenomena can be molecularly identified with airborne molecules, such as hydrocarbons or water molecules [37,38], adhering to surfaces over time. The result is the formation of an interface between the air and the "pure" surface that leads to a modification of the effective surface properties [12,39]. Molecules might permanently or semi-permanently adhere [39] and there may or may not be order and structure in such nanometric films, depending on the surface's atomic structure and composition [16] —even when the surface is immersed in water [12]. Over the past decade, our group has been mostly interested in the role of water adsorption and the resulting adsorption kinetics and corresponding variations on nanoscale forces $[27,34,35,40]$. Nanoscale forces can be investigated by means of force-distance profiles, force of adhesion maps, or other methods [33]. In particular, in Amplitude Modulation AFM (AM AFM), a dynamic mode of AFM operation, the standard observables, i.e., amplitude A, phase shift $\phi$, and cantilever separation $\mathrm{z}_{\mathrm{c}}$, can be turned into force distance curves (Figure 1a,b) by exploiting transformation algorithms [41-45]. The parameters that contain information about the cantilever and the environment, i.e., spring constant $k$, quality factor $Q$, and resonant frequency $f_{r}$, must be known. There are procedures to calibrate and exploit these parameters when employing force reconstruction methods [46]. The forces that the tip and surface exert on each other due to surface forces are typically termed tip-sample force $\mathrm{F}_{\mathrm{ts}}$, or simply $\mathrm{F}$ as in Figure 1, versus the distance $d$ force or curve. In Figure $1 b$, the force has been normalised and termed $\mathrm{F}^{*}$ by dividing the $\mathrm{F}$ by the absolute of the adhesion force $\mathrm{F}_{\mathrm{AD}}$. The adhesion force $\mathrm{F}_{\mathrm{AD}}$ is simply the minimum value of the force. All the data in Figure 1 are experimental data. 

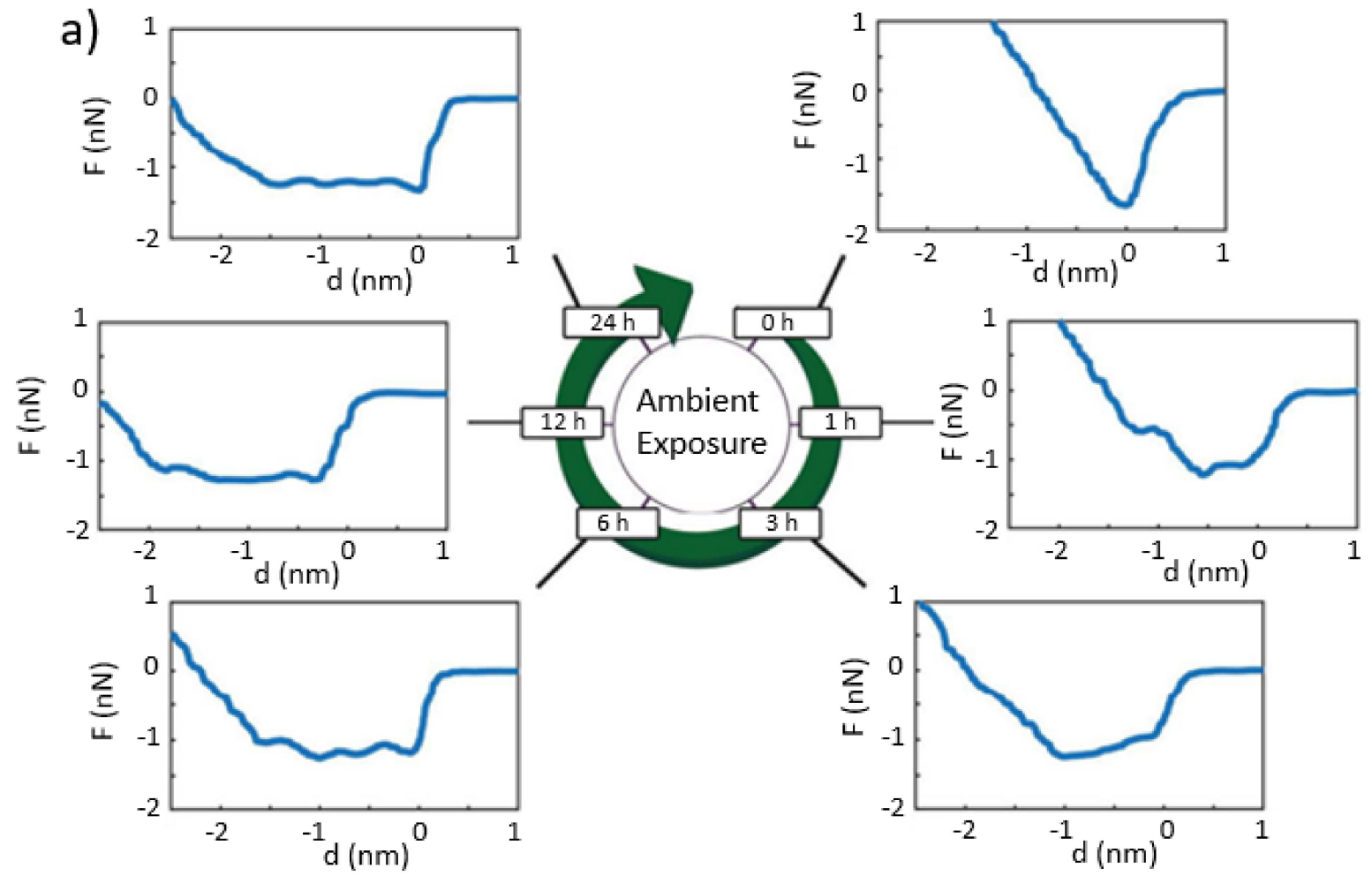

b)

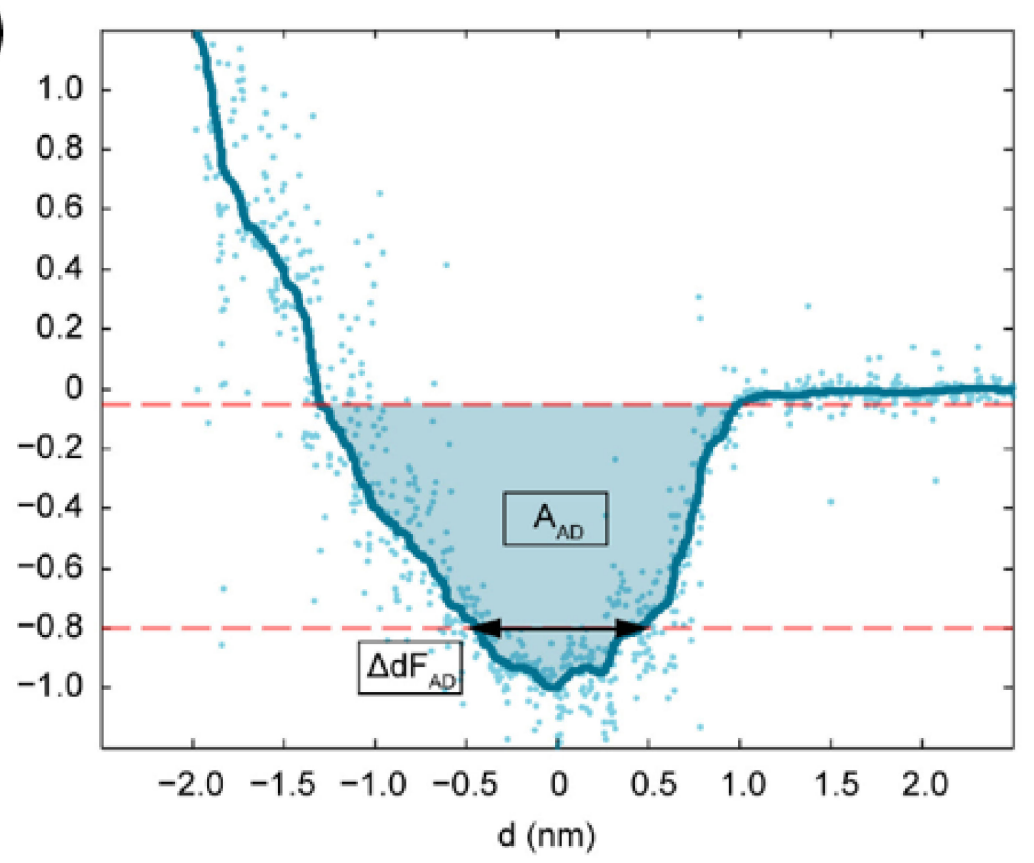

c)

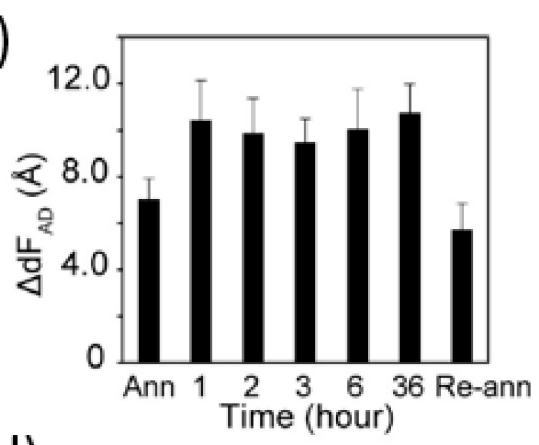

d)

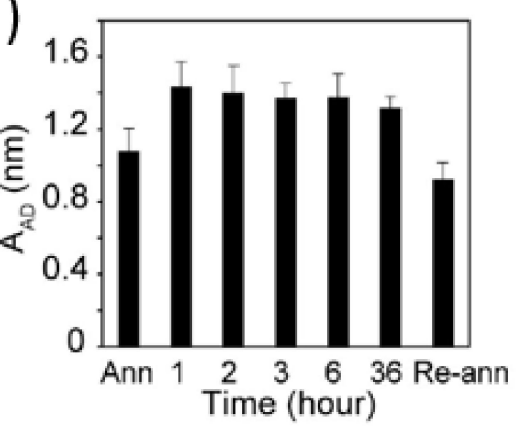

Figure 1. (a) Force versus distance curves obtained on a graphite sample as a function of time. The data have been acquired after cleaving the graphite $(0 \mathrm{~h}$, and $1 \mathrm{~h}, 3 \mathrm{~h}, 6 \mathrm{~h}, 12 \mathrm{~h}$ and $24 \mathrm{~h})$ by keeping the samples inside a controlled environment in between experiments (the temperature was relatively constant within the $23 \pm 2{ }^{\circ} \mathrm{C}$ interval and the relative humidity when acquiring the data were $\mathrm{RH} \sim 55 \pm 5 \%$ ). (b) Illustration of a normalized force versus distance curve obtained for a graphene/ $\mathrm{Cu}$ sample and highlighting the meaning of the parametrization of the curve via $\Delta \mathrm{dF}_{\mathrm{AD}}$ and the area under the curve $\mathrm{A}_{\mathrm{AD}}$. Mean values and standard deviations of (c) $\Delta \mathrm{dF}_{\mathrm{AD}}$ and (d) $\mathrm{A}_{\mathrm{AD}}$ for the graphene/Cu sample. (a) reprinted with permission from reference [34]. Copyright 2018 PCCP Owner Societies. (b-d) reprinted with permission from reference [47]. Copyright, 2014 ACS Ltd. 
Figure 1a illustrates how forces vary as samples, such as mica or graphite, are exposed to ambient air. The sample in Figure 1a is highly oriented pyrolytic graphite (HOPG) and the sample in Figure $1 \mathrm{~b}-\mathrm{d}$ is graphene/Cu. The details on sample preparation for the graphene/Cu sample can be found elsewhere [47]. The surfaces of mica and graphite are crystalline surfaces that have the advantage of being easily cleaved. In this way, these surfaces can be cleaved and immediately imaged, i.e., within minutes, or be exposed to ambient air or controlled RH (see Ref. [40]). The graphene/Cu samples can be imaged by first annealing at high temperatures under UHV conditions and then exposing the samples to controlled RH. The samples can be re-annealed after the experiments to reproduce the findings. The main feature to be observed in Figure 1a is a "plateau" that forms, i.e., a range of distances where the force reaches minima and is relatively independent of distance, as a function of time. In order to investigate the evolution of this plateau, the force can be parametrized (Figure $1 b$ ) by computing the distance of force of adhesion $\Delta \mathrm{dF}_{\mathrm{AD}}$ and the area under the curve $A_{A D}$. For more details, refer to the interpretation of these metrics in the literature [34,40]. The results obtained for the graphene/Cu sample (Figure 1c,d) show a similar behaviour to that of Figure $1 \mathrm{a}$ (graphite). In Figure 1c,d, the mean values and standard deviations are reported. Both parameters increase in the first hour after exposure to high $\mathrm{RH}$ and then drop after further annealing.

Clearly, the variations in force observed for the graphite sample in Figure 1a and for the graphene/ $\mathrm{Cu}$ sample in Figure $1 \mathrm{c}, \mathrm{d}$ induce a variation in cantilever dynamics for a set of operational parameters that also occurs in time. The implication is that the cantilever will behave very differently, and the surface will be probed very differently, in the first minutes after cleaving the sample in relation to hours later. A practical result for imaging is that different regimes of operation should become available with time. In our experience, after several hours of being exposed to the environment, the behaviour stabilizes. For this reason, the user must consider these variations and not expect that a given sample and setup will lead to certain results, irrespective of the dynamics of the "aging" process of the surface. In our work, we have only investigated the regimes of operation in two extreme regimes, i.e., freshly cleaved and aged after many hours, i.e., $24 \mathrm{~h}$ or more. An example is discussed with the help of Figure 2.

In Figure 2, two extremes are shown for a mica sample. In Figure 2a,b, the amplitude A (dashed black lines) and the minimum distance of approach $\mathrm{d}_{\mathrm{m}}$ (continuous black lines) are shown for an aged (Figure 2a), i.e., $\sim 24 \mathrm{~h}$, and a cleaved sample (Figure 2b), i.e., $\sim 0-1 \mathrm{~h}$. The minimum distance of approach per oscillation cycle can be obtained with the simple transform:

$$
\mathrm{d}_{\mathrm{m}}=\mathrm{z}_{\mathrm{c}}-\mathrm{A}
$$

where both the cantilever surface separation $\mathrm{z}_{\mathrm{C}}$ and $\mathrm{A}$ are known. Furthermore, while $\mathrm{z}_{\mathrm{C}}$ is a relative value, since the absolute distance to the surface is not known experimentally, the difference with $\mathrm{A}$ is not. Several extra features manifest in both signals in the aged sample. It is important to note that for sufficiently large values of $z_{c}$ or $d_{m} A$ is quasi-constant and it is identified with what is typically termed free amplitude or $A_{0}$. When $A=A_{0}$, the tip-sample interaction is negligible. In terms of the behaviour in Figure $2 a, b, A$ initially decreases with $\mathrm{z}_{\mathrm{c}}$, and $\mathrm{d}_{\mathrm{m}}$ is relatively independent of $\mathrm{z}_{\mathrm{c}}$-with A decreasing in both cases. This oscillation branch is typically termed an attractive, low-amplitude [48] branch or L-state. For the cleaved surface, only this branch is available when the free amplitude is small enough [31,49]. Nevertheless, for sufficiently small values of $A$, there is a region of negative slope (Figure 2a) in A and positive slope in $d_{m}$ [21]. This oscillation branch is not accessible for imaging in AM AFM, but has the advantage of driving the tip closer to the surface for small oscillation amplitudes, precisely because of the accentuated positive slope in $\mathrm{d}_{\mathrm{m}}$. According to our simulations [32,49], the tip penetrates the hydration layer until perpetual water contact is made, i.e., the tip is in perpetual contact and immersed in the hydration layer. At this point, the amplitude starts to decrease with decreasing $\mathrm{z}_{\mathrm{C}}$ (see peak in Figure 2a indicated by an arrow). This other branch is termed the SASS (Small Amplitude Small Set-point) branch [21]. However, it is important to realize that this oscillation branch 
is not accessible by simply setting a small amplitude. Rather, the hydration layer must be present, and the tip must go past the region of the negative slope. The first condition relies on the dynamics of hydration, and the second on operating the instrument with the understanding that several oscillation branches are available for a given set of operational parameters. In the SASS region, $\mathrm{d}_{\mathrm{m}}$ decreases with decreasing A, implying that the smaller $\mathrm{A}$ is, the less the tip indents the sample (Figure 2a). Thus, for high-resolution and minimally invasive imaging, $A$ or $A_{s p}$, where sp stands for the set point when imaging, must be set as small as possible. Nevertheless, experimentally, the implication is that $\mathrm{A}_{0}$ cannot be made arbitrarily small. Rather, in both AM and Frequency Modulation FM AFM, the drive amplitude must be increased in order to stabilize the tip. This is because $\mathrm{A}_{0}$ controls the energy input into the system; therefore, the stability of the dynamics is against the non-linear interaction and noise. The experimentally reconstructed force versus distance profile is shown for both cases in Figure 2c. For the aged case, the "plateau" is observed as discussed when considering Figure 1a. Figure 1d shows the behaviour of HOPG in terms of the absolute of the adhesion force $\left|\mathrm{F}_{\mathrm{AD}}\right|$ and the area under the curve $\mathrm{A}_{\mathrm{AD}}$. Here, $\left|\mathrm{F}_{\mathrm{AD}}\right|$ decreases rapidly in one hour and then remains relatively constant, but $\mathrm{A}_{\mathrm{AD}}$ keeps increasing up to $24 \mathrm{~h}$ after cleaving. Our interpretation is that molecular adsorption is taking place, even though it does not affect $\mathrm{F}_{\mathrm{AD}}$. This example illustrates that $\mathrm{F}_{\mathrm{AD}}$ alone is not enough to parametrize the evolution of the attractive force as a function of time. After the experiment, the HOPG sample was annealed. A relation in terms of $\mathrm{F}_{\mathrm{AD}}$ and contact angle is shown in Figure 2e for two cases; (1) when immediately probed after the cleaving case, (2) the case of being exposed to high $\mathrm{RH}$ for $24 \mathrm{~h}$, and (3) after annealing. The mean values and standard deviations for $\mathrm{F}_{\mathrm{AD}}$ are given for 15-30 data points. More details can be found in Ref. [40]. The procedure to obtain the contact angle is also described there, but a detailed explanation is outside the scope of this work.

Figure 3 is an illustration of the difference between imaging in (1) the attractive regime where the amplitudes $A_{s p}$ are comparable to $A_{0}$ (Figure 3a), (2) the SASS regime where $A_{\mathrm{sp}} / \mathrm{A}_{0}<<1$ (Figure $3 \mathrm{~b}$ ), and (3) the attractive regime where the condition $\mathrm{A}_{\mathrm{sp}} / \mathrm{A}_{0}<1$ also holds (Figure 3c). The inset in Figure 3d illustrates that the tip is fully immersed in the hydration layer when imaging in the SASS regime. The figure illustrates that the condition $A_{s p} / A_{0}<<1$ for a given $A_{0}$ can lead to different imaging regimes.

The reader can refer to the literature for a full description of the simulations describing the results for an amplitude A versus separation $z_{c}$ curve when inputting the forces into the simulations, such as those in Figures $1 \mathrm{a}$ and $2 \mathrm{c}$ for the aged case $[49,50]$. The simulation data allow to visualize a branch that is not observed in experimental Figure 2a, i.e., the repulsive regime or $\mathrm{H}$-state. Only where $\mathrm{A}_{0}$ is sufficiently large [51] can this branch be experimentally reached [2]. 

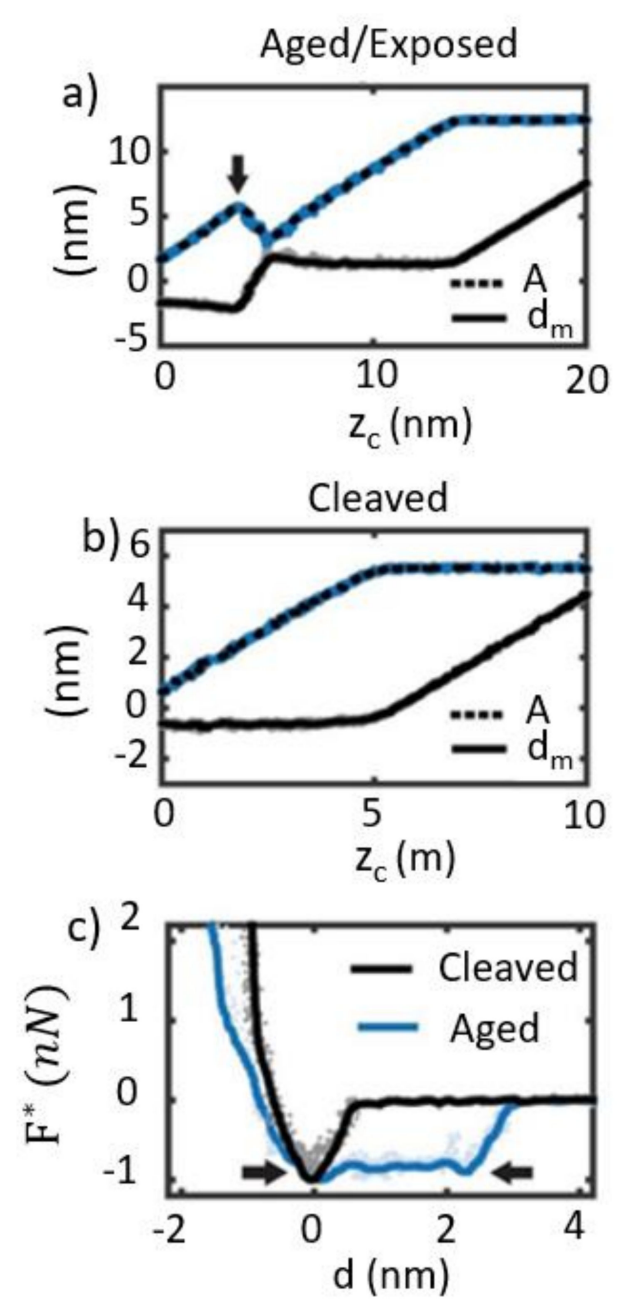

$\left|F_{A D}\right|$ in $(\mathrm{nN})$ and $\mathrm{A}_{\mathrm{AD}}$ in $(\mathrm{nNnm})$

d)

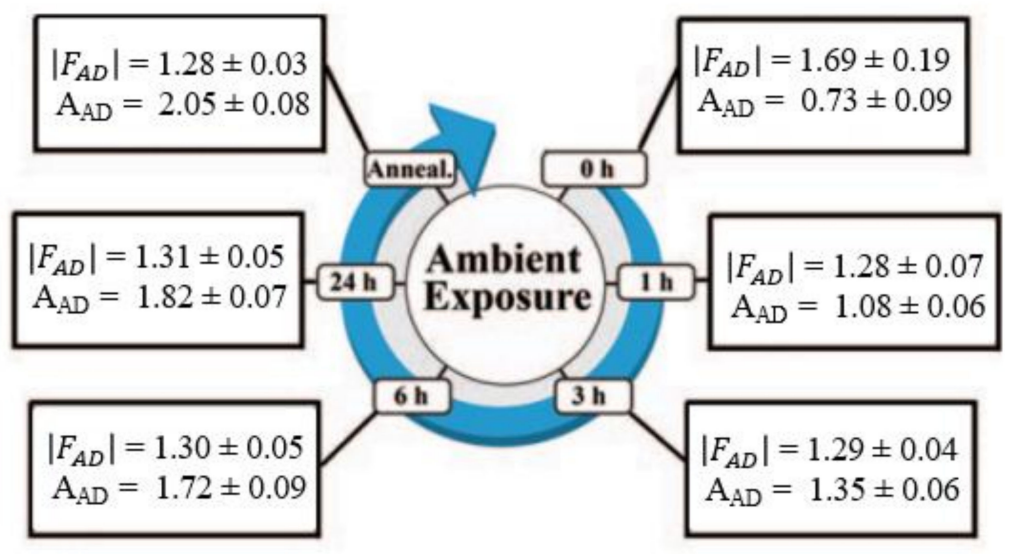

e)

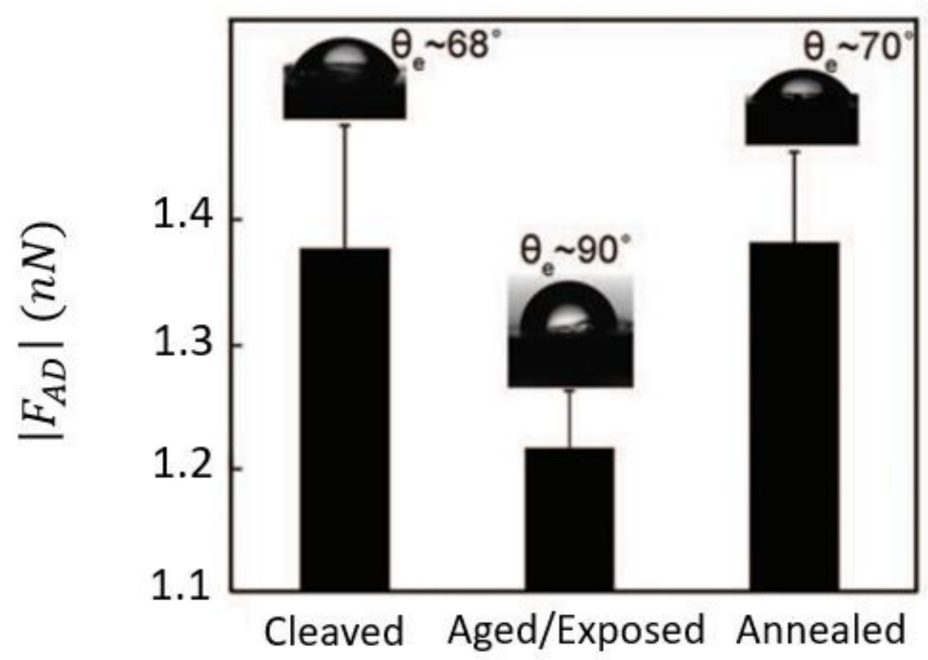

Figure 2. Amplitude $A$, separation $z_{c}$, and $d_{m}$ data for the (a) aged and (b) cleaved conditions for a mica sample. (c) $\mathrm{F}^{*}$ versus distance data, where the asterisk implies that the force has been normalized by dividing by the absolute of $\mathrm{F}_{\mathrm{AD}}$. This has been performed for convenience in order to visualise the difference in behaviour with $\mathrm{d}$ for both the cleaved (black lines) and the aged (blue lines) conditions. (d) Evolution of $\left|\mathrm{F}_{\mathrm{AD}}\right|$ and $\mathrm{A}_{\mathrm{AD}}$ with time of exposure in ambient conditions for an HOPG sample. (e) Evolution of the contact angle and $\left|\mathrm{F}_{\mathrm{AD}}\right|$ in three different cases for the HOPG sample. $(\mathbf{a}-\mathbf{c})$ reprinted, with permission from reference [31]. Copyright 2017 PCCP Owner Societies. (d,e) reprinted with permission from reference [40]. Copyright 2014 AIP Publishing. 
a) Attractive regime

b) Small amplitude SASS regime

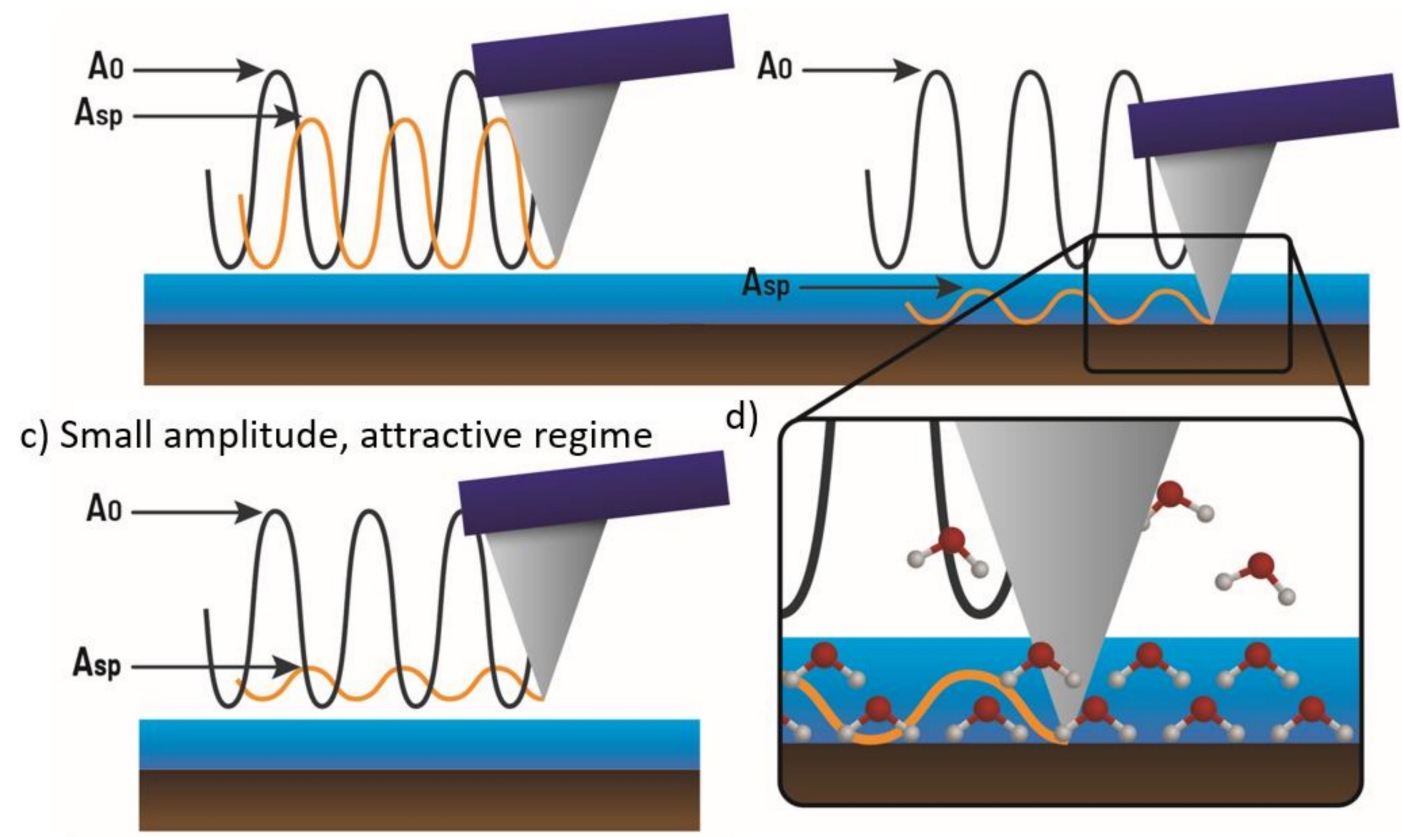

Figure 3. Illustration of a cantilever oscillating (a) above the hydration layer with amplitudes $\left(\mathrm{A}_{\mathrm{sp}}\right)$ comparable to $\mathrm{A}_{0}$ (standard attractive regime imaging), (b) with amplitudes $\mathrm{A}$ smaller than $\mathrm{A}_{0}$ when the tip is permanently immersed in the hydration layer (SASS regime), (c) above the hydration layer with small amplitudes $\left(\mathrm{A}_{\mathrm{sp}}\right)$ much smaller than $\mathrm{A}_{0}$ (Small amplitude attractive imaging). The inset in (d) is provided to illustrate that in the SASS regime the tip is immersed under the water layer where there might be structure and order. Airborne water molecules are also depicted for illustrative purposes.

\subsection{Other Possible Dynamic Parameters to Consider}

The definition of small amplitude is ambiguous because it depends on the cantilever, sample, and environmental parameters, i.e., when many parameters impose a limit on stability. One parameter that determines this limit is the tip radius R. Amongst others, the tip radius $\mathrm{R}$ controls the adhesion force $\mathrm{F}_{\mathrm{AD}}$ since $\mathrm{F}_{\mathrm{AD}} \equiv 4 \pi \gamma$, where $\gamma$ is the surface energy [39]. The larger the attractive forces, the stronger the pull-off force and the more energy is required for the system to reach the surface. In dynamic AFM, this means that larger values of $A_{0}$ are needed to reach the surface and the SASS imaging mode as $R$ increases. For this reason, $\mathrm{R}$ must be considered when defining what small amplitudes mean. The spring constant $k$ and the $Q$ factor might also play a role [52]. Since the tip radius $\mathrm{R}$ can vary during experiments, the user must consider the state of the tip together with the state of the hydration layer in order to image with small amplitudes. We have carried out some experiments [49] to understand the consequences of attempting to reach the SASS regime with a relatively blunt tip, i.e., $R>20 \mathrm{~nm}$. Nevertheless, these experiments were not systematic. It is likely that different force regimes are available for imaging, as surfaces also age as a function of $R$. This would be consistent with the fact that when $R$ is of the order of magnitude of the water film, the Kelvin equation and other macroscopic behaviours might not hold $[16,26]$. Finally, it is worth noting that in the SASS regime, the mean deflection $\mathrm{z}_{0}$ can be employed to map the adhesion force $\mathrm{F}_{\mathrm{AD}}$ while acquiring the standard topography. This is because the tip oscillates inside the hydration layer where the force is almost constant and approximately $\mathrm{F}_{\mathrm{AD}}$ [33]. The expression to map the adhesion force becomes a simple transformation of the mean deflection map:

$$
\mathrm{F}_{\mathrm{AD}}=\mathrm{kz}_{0}
$$

Incidentally, and practically, monitoring a step-in deflection could be used experimentally to discriminate between the attractive regime and the SASS regime. It is likely, 
however, that as a systematic investigation of force reconstruction, aging, and imaging regime is carried out, new identities or useful expressions will emerge. Finally, the SASS regime can also be accessed and exploited in multifrequency AFM [3,53]. In this regime, sample indentation $\delta$ is minimal but non-zero. It can also be shown that the small amplitudes, when imaging in SASS, can be exploited to produce expressions to quantify surface properties [33].

\section{Conclusions}

In this work, we have reviewed and discussed (1) force measurements in air as a function of time and (2) small-amplitude imaging in dynamic AFM in the presence and absence of water layers. The objective has been to connect and coordinate theoretical and experimental work from these two topics to establish the key problems that might emerge when imaging as a result of the multiple and variable force regimes available. On the one hand, force measurements on freshly created surfaces show that the force presents an attractive and a repulsive component, qualitatively similar in shape to the standard Lennard Jones [54] profile. On the other hand, as surfaces age and as they are exposed to airborne water molecules, water films emerge on surfaces and force profiles develop a "plateau" of constant force that ranges from 1 to $2 \mathrm{~nm}$, and that approximates the adhesion or minima in force. The work that we have carried out over the past decade has been discussed to show that this process is relevant over at least a few hours, depending on the surface, after the surface is created. Previously, we had shown that small-amplitude imaging, i.e., $\sim 0.1-1 \mathrm{~nm}$, for "aged" surfaces can lead to imaging in perpetual contact with the water layer while increasing resolution. Nevertheless, the possibility of imaging in conditions where (1) there are no water layers or (2) there are water layers is simplistic and binary. Rather, in agreement with the evolution of forces, the imaging regimes, and the way to reach them, should also be expected to be dynamic. The tip radius has also been shown to be a key variable when considering the dynamics of force regimes. In short, as AFM methods advance, it will become paramount to report not only operational, cantilever, and sample parameters, but also the state of the surface while imaging. The final point is that future research would benefit from coordinating the two lines of research that we have explored, i.e., force reconstruction and the emergence of imaging regimes as surfaces age.

Author Contributions: Conceptualization, S.S., T.A.O., C.-Y.L. and M.C.; methodology, S.S., T.A.O., C.-Y.L. and M.C.; investigation, S.S., T.A.O., C.-Y.L. and M.C.; writing-original draft preparation, S.S.; writing-review and editing, S.S., T.A.O., C.-Y.L. and M.C.; visualization, S.S.; supervision, S.S.; project administration, M.C.; funding acquisition, M.C. All authors have read and agreed to the published version of the manuscript.

Funding: This research received no external funding.

Acknowledgments: The authors acknowledge the support from the Arctic Centre for Sustainable Energy (ARC) at UiT-the Arctic University of Norway through Grant No. 310026. The illustration in Figure 3 has been produced by graphic designer Maritsa Kissamitaki.

Conflicts of Interest: The authors declare no conflict of interest.

\section{References}

1. Hansma, P.K.; Cleveland, J.P.; Radmacher, M.; Walters, D.A.; Hillner, P.E.; Bezanilla, M.; Fritz, M.; Vie, D.; Hansma, H.G.; Prater, C.B.; et al. Tapping mode atomic force microscopy in liquids. Appl. Phys. Lett. 1994, 64, 1738-1740. [CrossRef]

2. García, R.; Paulo, A.S. Attractive and repulsive tip-sample interaction regimes in tapping-mode atomic force microscopy. Phys. Rev. B 1999, 60, 4961-4967. [CrossRef]

3. Eichhorn, A.L.; Dietz, C. Simultaneous Deconvolution of In-Plane and Out-of-Plane Forces of HOPG at the Atomic Scale under Ambient Conditions by Multifrequency Atomic Force Microscopy. Adv. Mater. Interfaces 2021, 8, 2101288. [CrossRef]

4. Gauthier, M.; Pérez, R.; Arai, T.; Tomitori, M.; Tsukada, M. Interplay between Nonlinearity, Scan Speed, Damping, and Elec-tronics in Frequency Modulation Atomic-Force Microscopy. Phys. Rev. Lett. 2002, 89, 146104. [CrossRef]

5. Rana, S.; Pota, H.; Petersen, I.R. A Survey of Methods Used to Control Piezoelectric Tube Scanners in High-Speed AFM Imaging. Asian J. Control. 2018, 20, 1379-1399. [CrossRef]

6. García, R.; Perez, R. Dynamic atomic force microscopy methods. Surf. Sci. Rep. 2002, 47, 197-301. [CrossRef] 
7. Ortega-Esteban, A.; Gonzalez, F.; Martin, N.; de Fablo, P. Structural and mechanical characterization of viruses with AFM. In Atomic Force Microscopy. Methods in Molecular Biology; Humana Press: New York, NY, USA, 2019; Volume 1886, pp. $259-278$.

8. Garcia, R. Nanomechanical mapping of soft materials with the atomic force microscope: Methods, theory and applications. Chem. Soc. Rev. 2020, 49, 5850-5884. [CrossRef]

9. Ramos, J.R. Tip radius preservation for high resolution imaging in amplitude modulation atomic force microscopy. Appl. Phys. Lett. 2014, 105, 43111. [CrossRef]

10. Trinidad, E.R.; Gribnau, T.W.; Belardinelli, P.; Staufer, U.; Alijani, F. Nonlinear dynamics for estimating the tip radius in atomic force microscopy. Appl. Phys. Lett. 2017, 111, 123105. [CrossRef]

11. Hernández-Muñoz, J.; Uhlig, M.R.; Benaglia, S.; Chacón, E.; Tarazona, P.; García, R. Subnanometer Interfacial Forces in ThreeDimensional Atomic Force Microscopy: Water and Octane near a Mica Surface. J. Phys. Chem. C 2020, 124, 26296-26303. [CrossRef]

12. Uhlig, M.R.; Benaglia, S.; Thakkar, R.; Comer, J.; Garcia, R. Atomically resolved interfacial water structures on crystalline hy-drophilic and hydrophobic surfaces. Nanoscale 2021, 13, 5275-5283. [CrossRef]

13. Fukuma, T.; Ueda, Y.; Yoshioka, S.; Asakawa, H. Atomic-Scale Distribution of Water Molecules at the Mica-Water Interface Visualized by Three-Dimensional Scanning Force Microscopy. Phys. Rev. Lett. 2010, 104, 16101. [CrossRef]

14. Jiao, F.; Cannon, K.S.; Lin, Y.-C.; Gladfelter, A.S.; Scheuring, S. The hierarchical assembly of septins revealed by high-speed AFM. Nat. Commun. 2020, 11, 1-13. [CrossRef]

15. Stilp, F.; Bereczuk, A.; Berwanger, J.; Mundigl, N.; Richter, K.; Giessibl, F.J. Very weak bonds to artificial atoms formed by quantum corrals. Science 2021, 372, 1196-1200. [CrossRef]

16. Uhlig, M.R.; Garcia, R. In Situ Atomic-Scale Imaging of Interfacial Water under 3D Nanoscale Confinement. Nano Lett. 2021, 21, 5593-5598. [CrossRef]

17. Calò, A.; Robles, O.V.; Santos, S.; Verdaguer, A. Capillary and van der Waals interactions on CaF $\mathrm{C}_{2}$ crystals from amplitude modulation AFM force reconstruction profiles under ambient conditions. Beilstein J. Nanotechnol. 2015, 6, 809-819. [CrossRef]

18. Lacasa, J.S.; Almonte, L.; Colchero, J. In situ characterization of nanoscale contaminations adsorbed in air using atomic force microscopy. Beilstein J. Nanotechnol. 2018, 9, 2925-2935. [CrossRef]

19. Wastl, D.S.; Weymouth, A.; Giessibl, F. Optimizing atomic resolution of force microscopy in ambient conditions. Phys. Rev. B 2013, 87, 245415. [CrossRef]

20. Giessibl, F.J. AFM's path to atomic resolution. Mater. Today 2005, 8, 32-41. [CrossRef]

21. Santos, S.; Barcons, V.; Christenson, H.K.; Billingsley, D.J.; Bonass, W.A.; Font, J.; Thomson, N.H. Stability, resolution, and ultra-low wear amplitude modulation atomic force microscopy of DNA: Small am-plitude small set-point imaging. Appl. Phys. Lett. 2013, 103, 63702-63705. [CrossRef]

22. Zitzler, L.; Herminghaus, S.; Mugele, F. Capillary forces in tapping mode atomic force microscopy. Phys. Rev. B 2002, 66, 155436. [CrossRef]

23. Leroch, S.; Wendland, M. Influence of Capillary Bridge Formation onto the Silica Nanoparticle Interaction Studied by Grand Canonical Monte Carlo Simulations. Langmuir 2013, 29, 12410-12420. [CrossRef] [PubMed]

24. Sahagún, E.; García-Mochales, P.; Sacha, G.; Sáenz, J.J. Energy dissipation due to capillary interactions: Hydrophobicity maps in force microscopy. Phys. Rev. Lett. 2007, 98, 176106. [CrossRef]

25. Yaminsky, V. The hydrophobic force: The constant volume capillary approximation. Colloids Surf. A Physicochem. Eng. Asp. 1999, 159, 181-195. [CrossRef]

26. Yang, Q.; Sun, P.Z.; Fumagalli, L.; Stebunov, Y.V.; Haigh, S.J.; Zhou, Z.W.; Grigorieva, I.V.; Wang, F.C.; Geim, A.K. Capillary condensation under atomic-scale confinement. Nature 2020, 588, 250-253. [CrossRef]

27. Amadei, C.A.; Tang, T.C.; Chiesa, M.; Santos, S. The aging of a surface and the evolution of conservative and dissipative na-noscale interactions. J. Chem. Phys. 2013, 139, 84708. [CrossRef]

28. Klasen, A.; Baumli, P.; Sheng, Q.; Johannes, E.; Bretschneider, S.A.; Hermes, I.M.; Bergmann, V.W.; Gort, C.; Axt, A.; Weber, S.A.L.; et al. Removal of Surface Oxygen Vacancies Increases Conductance Through $\mathrm{TiO}_{2}$ Thin Films for Perovskite Solar Cells. J. Phys. Chem. 2019, 123, 13458-13466. [CrossRef]

29. Zhang, Y.; Zhu, X.; Chu, C.; Xiao, X.; Chen, B. Applications of atomic force microscopy-based imaging and force spectroscopy in assessing environmental interfacial processes. Crit. Rev. Environ. 2021, 1-32. [CrossRef]

30. Lu, J.-Y.; Lai, C.-Y.; Almansoori, I.; Chiesa, M. The evolution in graphitic surface wettability with first-principles quantum simulations: The counterintuitive role of water. Phys. Chem. Chem. Phys. 2018, 20, 22636-22644. [CrossRef]

31. Alshehhi, M.; Alhassan, S.M.; Chiesa, M. Dependence of surface aging on DNA topography investigated in attractive bimodal atomic force microscopy. Phys. Chem. Chem. Phys. 2017, 19, 10231-10236. [CrossRef]

32. Santos, S. Enhanced sensitivity and contrast with bimodal atomic force microscopy with small and ultra-small amplitudes in ambient conditions. Appl. Phys. Lett. 2013, 103, 231603. [CrossRef]

33. Lai, C.-Y.; Santos, S.; Chiesa, M. Systematic Multidimensional Quantification of Nanoscale Systems from Bimodal Atomic Force Microscopy Data. ACS Nano 2016, 10, 6265-6272. [CrossRef]

34. Chiesa, M.; Lai, C.-Y. Surface aging investigation by means of an AFM-based methodology and the evolution of conservative nanoscale interactions. Phys. Chem. Chem. Phys. 2018, 20, 19664-19671. [CrossRef] 
35. Santos, S.; Amadei, C.A.; Lai, C.-Y.; Olukan, T.; Lu, J.-Y.; Font, J.; Barcons, V.; Verdaguer, A.; Chiesa, M. Investigating the Ubiquitous Presence of Nanometric Water Films on Surfaces. J. Phys. Chem. C 2021, 125, 15759-15772. [CrossRef]

36. Giessibl, F.J. Advances in atomic force microscopy. Rev. Mod. Phys. 2003, 75, 949-983. [CrossRef]

37. Verdaguer, A.; Sacha, G.M.; Bluhm, A.H.; Salmeron, M. Molecular Structure of Water at Interfaces: Wetting at the Nanometer Scale. Chem. Rev. 2006, 106, 1478-1510. [CrossRef]

38. Henderson, M. The interaction of water with solid surfaces: Fundamental aspects revisited. Surf. Sci. Rep. 2002, 46, 1-308. [CrossRef]

39. Israelachvili, J.N. Intermolecular and Surface Forces; Elsevier Academic Press: London, UK, 2005.

40. Amadei, C.A.; Lai, C.-Y.; Heskes, D.; Chiesa, M. Time dependent wettability of graphite upon ambient exposure: The role of water adsorption. J. Chem. Phys. 2014, 141, 84709. [CrossRef]

41. Sader, J.E.; Jarvis, S.P. Accurate formulas for interaction force and energy in frequency modulation force spectroscopy. Appl. Phys. Lett. 2004, 84, 1801-1803. [CrossRef]

42. Katan, A.; Van Es, M.H.; Oosterkamp, T.H. Quantitative force versus distance measurements in amplitude modulation AFM: A novel force inversion technique. Nanotechnology 2009, 20, 165703. [CrossRef]

43. Amir, F.P.; Martin-Jimenez, D.; Garcia, R. Force reconstruction from tapping mode force microscopy experiments. Nanotechnology 2015, 26, 185706.

44. Hu, S.; Raman, A. Inverting amplitude and phase to reconstruct tip-sample interaction forces in tapping mode atomic force microscopy. Nanotechnology 2008, 19, 375704. [CrossRef] [PubMed]

45. Huber, F.; Giessibl, F. Experimental demonstration of pitfalls and remedies for precise force deconvolution in frequencymodulation atomic force microscopy. arXiv 2020, arXiv:2001.11323.

46. Sader, J.E.; Sanelli, J.A.; Adamson, B.; Monty, J.; Wei, X.; Crawford, S.A.; Friend, J.; Marusic, I.; Mulvaney, P.; Bieske, E. Spring constant calibration of atomic force microscope cantilevers of arbitrary shape. Rev. Sci. Instrum. 2012, 83, 103705. [CrossRef]

47. Lai, C.-Y.; Tang, T.-C.; Amadei, C.A.; Marsden, A.J.; Verdaguer, A.; Wilson, N.; Chiesa, M. A nanoscopic approach to studying evolution in graphene wettability. Carbon 2014, 80, 784-792. [CrossRef]

48. García, R.; Paulo, A.S. Dynamics of a vibrating tip near or in intermittent contact with a surface. Phys. Rev. B 2000, 61, R13381-R13384. [CrossRef]

49. Santos, S. Dynamic Atomic Force Microscopy and Applications in Biomolecular Imaging; University of Leeds: Leeds, UK, 2011. Available online: https:/ / etheses.whiterose.ac.uk/1910/ (accessed on 7 October 2021).

50. Barcons, V.; Santos, S.; Bonass, W.; Font, J.; Thomson, N.H. Mono-stability of sharp tips interacting with surface hydration layers. arXiv 2015, arXiv:1506.03961.

51. Santos, S.; Guang, L.; Souier, T.; Gadelrab, K.; Chiesa, M.; Thomson, N.H. A method to provide rapid in situ determination of tip radius in dynamic atomic force microscopy. Rev. Sci. Instrum. 2012, 83, 43707. [CrossRef]

52. Santos, S.; Barcons, V.; Font, J.; Thomson, N.H. Bi-stability of amplitude modulation AFM in air: Deterministic and stochastic outcomes for imaging biomolecular systems. Nanotechnology 2010, 21, 225710. [CrossRef]

53. Santos, S. Phase contrast and operation regimes in multifrequency atomic force microscopy. Appl. Phys. Lett. 2014, 104, 143109. [CrossRef]

54. Wang, X.; Ramírez-Hinestrosa, S.; Dobnikar, J.; Frenkel, D. The Lennard-Jones potential: When (not) to use it. Phys. Chem. Chem. Phys. 2020, 22, 10624-10633. [CrossRef] 